\title{
New developments in the spectral asymptotics of quantum gravity
}

\author{
Giampiero Esposito†, Guglielmo Fucci $\ddagger$, Alexander Yu \\ Kamenshchik§, Klaus Kirsten\| \\ $\nmid \mathrm{INFN}$, Sezione di Napoli, and Dipartimento di Scienze Fisiche, Complesso \\ Universitario di Monte S. Angelo, Via Cintia, Edificio N', 80126 Napoli, Italy \\ $\ddagger$ Department of Physics, New Mexico Institute of Mining and Technology, Leroy \\ Place 801, Socorro, NM 87801, USA \\ $\S$ Dipartimento di Fisica and INFN, Sezione di Bologna, Via Irnerio 46, 40126 \\ Bologna, Italy; L D Landau Institute for Theoretical Physics, Kosygin str. 2, 119334 \\ Moscow, Russia \\ || Department of Mathematics, Baylor University, Waco, TX 76798, USA
}

\begin{abstract}
A vanishing one-loop wave function of the Universe in the limit of small three-geometry is found, on imposing diffeomorphism-invariant boundary conditions on the Euclidean 4-ball in the de Donder gauge. This result suggests a quantum avoidance of the cosmological singularity driven by full diffeomorphism invariance of the boundary-value problem for one-loop quantum theory. All of this is made possible by a peculiar spectral cancellation on the Euclidean 4-ball, here derived and discussed.
\end{abstract}

\section{Introduction}

Since the early eighties there has been a substantial revival of interest in quantum cosmology, motivated by the hope of obtaining a complete picture of how the universe could arise and evolve 1, 2, 3, 4. By complete we here mean a theoretical description where, by virtue of the guiding principles of physics and mathematics, both the differential equations of the theory and the associated boundary (and initial) conditions are fully specified. Even though modern theoretical cosmology deals with yet other deep issues such as dark matter, dark energy [5, 6] and cosmic strings [7, the effort of formulating the appropriate boundary conditions for the quantum state of the universe [8, or at least for its (one-loop) semiclassical approximation, plays again a key role, since the universe might have had a semiclassical origin [9], and the various orders in $\hbar$ in the loop expansion describe the departure from the underlying classical dynamics.

The physical motivations of our research result therefore from the following active areas of research:

(i) Functional integrals and space-time approach to quantum field theory [10].

(ii) Attempt to derive the whole set of physical laws from invariance principles [11. 
(iii) How to derive the early universe evolution from quantum physics; how to make sense of a wave function of the universe and of Hartle-Hawking quantum cosmology [12, 8.

(iv) Spectral theory and its physical applications, including functional determinants in one-loop quantum theory and hence the first corrections to classical dynamics [13].

The boundary conditions that we study are part of a unified scheme for Maxwell, Yang-Mills and Quantized General Relativity at one loop, i.e. [14]

$$
\begin{aligned}
& {[\pi \mathcal{A}]_{\mathcal{B}}=0,} \\
& {[\Phi(A)]_{\mathcal{B}}=0,} \\
& {[\varphi]_{\mathcal{B}}=0 .}
\end{aligned}
$$

With our notation, $\pi$ is a projector acting on the gauge field $\mathcal{A}, \Phi$ is the gauge-fixing functional, $\varphi$ is the ghost field (or full set of ghost fields) [15. Both equation (1) and (2) are preserved under infinitesimal gauge transformations provided that the ghost obeys homogeneous Dirichlet conditions as in (3). For gravity, we choose $\Phi$ so as to have an operator $P$ of Laplace type on metric perturbations in the one-loop Euclidean theory.

\section{Eigenvalue conditions for scalar modes}

On the Euclidean 4-ball, we expand metric perturbations $h_{\mu \nu}$ in terms of scalar, transverse vector, transverse-traceless tensor harmonics on $S^{3}$. For vector, tensor and ghost modes, boundary conditions reduce to Dirichlet or Robin [16]. For scalar modes, one finds eventually the eigenvalues $E=x^{2}$ from the roots $x$ of [16]

$$
\begin{aligned}
& J_{n}^{\prime}(x) \pm \frac{n}{x} J_{n}(x)=0, \\
& J_{n}^{\prime}(x)+\left(-\frac{x}{2} \pm \frac{n}{x}\right) J_{n}(x)=0 .
\end{aligned}
$$

Note that both $x$ and $-x$ solve the same equation. For example, at small $n$ and large $x$, the roots of Eq. (5) with $+\operatorname{sign}$ in front of $\frac{n}{x} \operatorname{read}$ as (here $\left.s=0,1, \ldots, \infty\right)$

$$
x(s, n) \sim \beta(s, n)\left[1+\frac{\gamma_{1}}{\beta^{2}(s, n)}+\frac{\gamma_{2}}{\beta^{4}(s, n)}+\frac{\gamma_{3}}{\beta^{6}(s, n)}+\mathrm{O}\left(\beta^{-8}\right)\right],
$$

where

$$
\beta(s, n) \equiv \pi\left(s+\frac{n}{2}+\frac{3}{4}\right),
$$

and (having defined $m \equiv 4 n^{2}$ )

$$
\begin{aligned}
& \gamma_{1}(m) \equiv-\frac{(m-17)}{8} \\
& \gamma_{2}(m) \equiv-\frac{3455}{384}+2 m^{1 / 2}+\frac{67}{192} m-\frac{7}{384} m^{2}, \\
& \gamma_{3}(m)=\frac{1117523}{15360}-\frac{115}{4} m^{1 / 2}-\frac{5907}{5120} m+\frac{3}{4} m^{3 / 2}+\frac{421}{3072} m^{2}-\frac{83}{15360} m^{3},
\end{aligned}
$$

as we have found in the second item of [17. 


\section{Four generalized $\zeta$-functions for scalar modes}

From Eqs. (4) and (5) we obtain the following integral representations of the resulting $\zeta$-functions upon exploiting the Cauchy theorem and rotation of contour [16, 17]:

$$
\zeta_{A, B}^{ \pm}(s) \equiv \frac{(\sin \pi s)}{\pi} \sum_{n=3}^{\infty} n^{-(2 s-2)} \int_{0}^{\infty} d z z^{-2 s} \frac{\partial}{\partial z} \log F_{A, B}^{ \pm}(z n),
$$

where (here $\left.\beta_{+} \equiv n, \beta_{-} \equiv n+2\right)$

$$
\begin{aligned}
& F_{A}^{ \pm}(z n) \equiv z^{-\beta_{ \pm}}\left(z n I_{n}^{\prime}(z n) \pm n I_{n}(z n)\right), \\
& F_{B}^{ \pm}(z n) \equiv z^{-\beta_{ \pm}}\left(z n I_{n}^{\prime}(z n)+\left(\frac{(z n)^{2}}{2} \pm n\right) I_{n}(z n)\right),
\end{aligned}
$$

$I_{n}$ being the modified Bessel functions of first kind. Regularity at the origin is easily proved in the elliptic sectors, corresponding to $\zeta_{A}^{ \pm}(s)$ and $\zeta_{B}^{-}(s)$.

\section{Regularity of $\zeta_{B}^{+}$at $s=0$}

We now define $\tau \equiv\left(1+z^{2}\right)^{-1 / 2}$ and consider the uniform asymptotic expansion (away from $\tau=1$, with notation as in [16, 17])

$$
z^{\beta_{+}} F_{B}^{+}(z n) \sim \frac{\mathrm{e}^{n \eta(\tau)}}{h(n) \sqrt{\tau}} \frac{\left(1-\tau^{2}\right)}{\tau}\left(1+\sum_{j=1}^{\infty} \frac{r_{j,+}(\tau)}{n^{j}}\right),
$$

the functions $r_{j,+}$ being obtained from the Olver polynomials for the uniform asymptotic expansion of $I_{n}$ and $I_{n}^{\prime}$ [18. On splitting $\int_{0}^{1} d \tau=\int_{0}^{\mu} d \tau+\int_{\mu}^{1} d \tau$ with $\mu$ small, we get an asymptotic expansion of the l.h.s. by writing, in the first interval on the r.h.s.,

$$
\log \left(1+\sum_{j=1}^{\infty} \frac{r_{j,+}(\tau)}{n^{j}}\right) \sim \sum_{j=1}^{\infty} \frac{R_{j,+}(\tau)}{n^{j}}
$$

and then computing

$$
C_{j}(\tau) \equiv \frac{\partial R_{j,+}}{\partial \tau}=(1-\tau)^{-j-1} \sum_{a=j-1}^{4 j} K_{a}^{(j)} \tau^{a}
$$

The integral $\int_{\mu}^{1} d \tau$ is instead found to yield a vanishing contribution in the $\mu \rightarrow 1$ limit (second item in [17]). Remarkably, by virtue of the spectral identity

$$
g(j) \equiv \sum_{a=j}^{4 j} \frac{\Gamma(a+1)}{\Gamma(a-j+1)} K_{a}^{(j)}=0,
$$

which holds $\forall j=1, \ldots, \infty$, we find

$$
\lim _{s \rightarrow 0} s \zeta_{B}^{+}(s)=\frac{1}{6} \sum_{a=3}^{12} a(a-1)(a-2) K_{a}^{(3)}=0,
$$


and

$$
\zeta_{B}^{+}(0)=\frac{5}{4}+\frac{1079}{240}-\frac{1}{2} \sum_{a=2}^{12} \omega(a) K_{a}^{(3)}+\sum_{j=1}^{\infty} f(j) g(j)=\frac{296}{45}
$$

where

$$
\begin{aligned}
\omega(a) & \equiv \frac{1}{6} \frac{\Gamma(a+1)}{\Gamma(a-2)}\left[-\log (2)-\frac{\left(6 a^{2}-9 a+1\right)}{4} \frac{\Gamma(a-2)}{\Gamma(a+1)}\right. \\
& +2 \psi(a+1)-\psi(a-2)-\psi(4)], \\
f(j) & \equiv \frac{(-1)^{j}}{j !}\left[-1-2^{2-j}+\zeta_{R}(j-2)\left(1-\delta_{j, 3}\right)+\gamma \delta_{j, 3}\right] .
\end{aligned}
$$

The spectral cancellation (17) achieves three goals: (i) Vanishing of $\log 2$ coefficient in Eq. (19); (ii) Vanishing of $\sum_{j=1}^{\infty} f(j) g(j)$ in Eq. (19); (iii) Regularity at the origin of $\zeta_{B}^{+}$.

To cross-check our analysis, we evaluate $r_{j,+}(\tau)-r_{j,-}(\tau)$ and hence obtain $R_{j,+}(\tau)-R_{j,-}(\tau)$ for all $j$. Only $j=3$ contributes to $\zeta_{B}^{ \pm}(0)$, and we find

$$
\begin{aligned}
\zeta_{B}^{+}(0) & =\zeta_{B}^{-}(0)-\frac{1}{24} \sum_{l=1}^{4} \frac{\Gamma(l+1)}{\Gamma(l-2)}\left[\psi(l+2)-\frac{1}{(l+1)}\right] \kappa_{2 l+1}^{(3)} \\
& =\frac{206}{45}+2=\frac{296}{45}
\end{aligned}
$$

in agreement with Eq. (19), where $\kappa_{2 l+1}^{(3)}$ are the four coefficients on the right-hand side of

$$
\frac{\partial}{\partial \tau}\left(R_{3,+}-R_{3,-}\right)=\left(1-\tau^{2}\right)^{-4}\left(80 \tau^{3}-24 \tau^{5}+32 \tau^{7}-8 \tau^{9}\right) .
$$

Within this framework, the spectral cancellation reads as

$$
\sum_{l=1}^{4} \frac{\Gamma(l+1)}{\Gamma(l-2)} \kappa_{2 l+1}^{(3)}=0
$$

which is a particular case of

$$
\sum_{a=a_{\min }(j)}^{a=a_{\max }(j)} \frac{\Gamma((a+1) / 2)}{\Gamma((a+1) / 2-j)} \kappa_{a}^{(j)}=0 .
$$

Interestingly, the full $\zeta(0)$ value for pure gravity (i.e. including the contribution of tensor, vector, scalar and ghost modes) is then found to be positive: $\zeta(0)=\frac{142}{45}$ [17, which suggests a quantum avoidance of the cosmological singularity driven by full diffeomorphism invariance of the boundary-value problem for one-loop quantum theory [17. 


\section{Open problems}

Several open problems should be brought to the attention of the reader, and are as follows.

(i) We have encountered a boundary-value problem where the generalized $\zeta$-function remains well defined, even though the Mellin transform relating $\zeta$-function to heat kernel does not exist (see further comments below), since strong ellipticity is violated [14] (see also [19]). Are the spectral cancellations (17) and (25) a peculiar property of the Euclidean 4-ball, or can they be extended to more general Riemannian manifolds with non-empty boundary?

(ii) What is the deeper underlying reason for finding $\zeta_{B}^{+}(0)-\zeta_{B}^{-}(0)=2$ ? Is it possible to foresee a geometrical or topological or group-theoretical origin of this result?

(iii) Is it correct to say that our positive $\zeta(0)$ value for pure gravity engenders a quantum avoidance of the cosmological singularity at one-loop level? [17, 9] Does the result remain true in higher-loop calculations or on using other regularization techniques for the oneloop correction?

(iv) The whole scheme might be relevant for AdS/CFT in light of a profound link between AdS/CFT and the Hartle-Hawking wave function of the universe [20].

(v) What happens if one considers instead non-local boundary data, e.g. those giving rise to surface states for the Laplacian? [11, 21, 22]

As far as item (i) is concerned, we should add what follows. The integral representation (11) of the generalized $\zeta$-function is legitimate because, for any fixed $n$, there is a countable infinity of roots $x_{j}$ and $-x_{j}$ of Eqs. (4) and (5), and they grow approximately linearly with the integer $j$ counting such roots. The functions $F_{A}^{ \pm}$and $F_{B}^{ \pm}$ admit therefore a canonical-product representation [23] which ensures that the integral representation (11) reproduces the standard definition of generalized $\zeta$-function [16]. Furthermore, even though the Mellin transform relating $\zeta$-function to integrated heat kernel cannot be exploited when strong ellipticity is not fulfilled, it remains possible to define a generalized $\zeta$-function. For this purpose, a weaker assumption provides a sufficient condition, i.e. the existence of a sector in the complex plane free of eigenvalues of the leading symbol of the differential operator under consideration [16, 17. To make sure we have not overlooked some properties of the spectrum, we have been looking for negative eigenvalues or zero-modes, but finding none. Indeed, negative eigenvalues $E$ would imply purely imaginary roots $x=i y$ of Eq. (5), but such roots do not exist, as one can check both numerically and analytically; zero-modes would be non-trivial eigenfunctions belonging to zero-eigenvalues, but all modes (tensor, vector, scalar and ghost modes) are combinations of regular Bessel functions [16] (since we require regularity at the origin of the left-hand side of Eqs. (1)-(3)) for which this is

impossible. As far as we can see, we still find sources of singularities at the origin in 
the generalized $\zeta$-function resulting from lack of strong ellipticity, but the particular symmetries of the Euclidean 4-ball background reduce them to the four terms in Eq. (24), which add up to zero despite two of them are non-vanishing.

We have proposed to interpret the result $\zeta(0)=\frac{142}{45}$ for pure gravity as an indication that full diffeomorphism invariance of the boundary-value problem engenders a quantum avoidance of the cosmological singularity. Indeed, on the one hand, the work by Schleich 24] had found that, on restricting the functional integral to transverse-traceless perturbations, the one-loop semiclassical approximation to the wave function of the universe diverges at small volumes, at least for the boundary geometry of a three-sphere. The divergence of the wave functional does not imply, by itself, that the probability density of the wave functional diverges at small volumes, since the probability density $p[h]$ on the space of wave functionals $\psi[h]$ is given by $p[h]=m[h]|\psi|^{2}[h]$, where $m[h]$ is the measure on this space, the scaling of which is not known in general. On the other hand, in our manifestly covariant evaluation of the one-loop functional integral for the wave function of the universe, it seems incorrect to assume that the measure $m[h]$ scales in such a way as to cancel exactly the contribution of the squared modulus of $\psi$, which is proportional to the three-sphere radius raised to the power $2 \zeta(0)$. Thus, we find that our one-loop wave function of the universe vanishes at small volume. The normalizability condition of the wave function in the limit of small three-geometry, which is weaker than requiring it should vanish in this limit, was instead formulated and studied in [25].

The years to come will hopefully tell us whether our calculations may be viewed as a first step towards finding under which conditions a quantum theory of gravity is singularity free in cosmology [26]. For this purpose, it might also be interesting to study diffeomorphism-invariant boundary conditions for $f(R)$ theories of gravity, recently studied at one-loop level on manifolds without boundary [27.

\section{Acknowledgments}

We are grateful to Gerald Dunne, Dmitri Fursaev, Mariel Santangelo and Antonello Scardicchio for stimulating questions, and to Emilio Elizalde and Sergei Odintsov for having organized such a beautiful Conference. The work of G Esposito has been partially supported by PRIN SINTESI. K Kirsten is grateful to the Baylor University Research Committee, to the Max-Planck-Institute for Mathematics in the Sciences (Leipzig, Germany) and to the INFN for financial support. The work of A Yu Kamenshchik was partially supported by the Russian Foundation for Basic Research under the Grant No 02-02-16817 and by the Scientific School Grant No 2338.2003.2

\section{References}

[1] Hawking S W 1982 Pont. Acad. Sci. Scr. Varia 48563

[2] Vilenkin A 1984 Phys. Rev. D30 509

[3] Vilenkin A 1986 Phys. Rev. D33 3560

[4] Vilenkin A 1988 Phys. Rev. D37 888 
[5] Kamenshchik A Yu, Moschella U and Pasquier V 2001 Phys. Lett. B511 265

[6] Capozziello S, Nojiri S and Odintsov S D 2005 Unified phantom cosmology: inflation, dark energy and dark matter under the same standard (hep-th/0507182)

[7] Vilenkin A 2005 Cosmic strings: progress and problems (hep-th/0508135)

[8] Hawking S W 1984 Nucl. Phys. B239 257

[9] Hawking S W 2005 Phys. Scripta T117 49

[10] DeWitt B S 2003 The Global Approach to Quantum Field Theory (Clarendon Press, Oxford)

[11] Esposito G 2000 Int. J. Mod. Phys. A15 4539

[12] Hartle J B and Hawking S W 1983 Phys. Rev. D28 2960

[13] Esposito G 1994 Quantum Gravity, Quantum Cosmology and Lorentzian Geometries, Lecture Notes in Physics, New Series m, Vol. m12 (Springer, Berlin)

[14] Avramidi I G and Esposito G 1999 Commun. Math. Phys. 200495

[15] Esposito G, Kamenshchik A Yu and Pollifrone G 1997 Euclidean Quantum Gravity on Manifolds with Boundary, Vol. 85 of Fundamental Theories of Physics (Kluwer, Dordrecht)

[16] Esposito G, Fucci G, Kamenshchik A Yu and Kirsten K 2005 Class. Quantum Grav. 22957

[17] Esposito G, Fucci G, Kamenshchik A Yu and Kirsten K 2005 (hep-th/0506223 JHEP 0509:063)

[18] Olver F W J 1954 Phil. Trans. R. Soc. Lond. A 247328

[19] Dowker J S and Kirsten K 1997 Class. Quantum Grav. 14 L169

[20] Horowitz G T and Maldacena J 2004 JHEP 0402008

[21] Schröder M 1989 Rep. Math. Phys. 27259

[22] Esposito G 1999 Class. Quantum Grav. 161113

[23] Ahlfors L V 1966 Complex Analysis (New York: McGraw-Hill)

[24] Schleich K 1985 Phys. Rev. D32 1889

[25] Barvinsky A O and Kamenshchik A Yu 1990 Class. Quantum Grav. 7 L181

[26] Kiefer C 2005 Quantum gravity: general introduction and recent developments (gr-qc/0508120)

[27] Cognola G, Elizalde E, Nojiri S, Odintsov S D and Zerbini S 2005 JCAP 0502:010 